\title{
MATERIAS PRIMAS LEJANAS. EXPLOTACIÓN DE RECURSOS LÍTICOS NO LOCALES PARA LA CONFECCIÓN DE MICRO-RASPADORES EN EL CENTRO DE TIERRA DEL FUEGO: EL CASO ARQUEOLÓGICO KAMI ${ }^{1}$
}

HERNÁN DE ANGELIS*

\begin{abstract}
RESUMEN
El objetivo del trabajo es presentar los resultados obtenidos a partir del estudio de un conjunto de micro-raspadores provenientes del sitio arqueológico Kami 1, ubicado en la margen sur del lago Fagnano. Este conjunto presenta como característica sobresaliente un porcentaje elevado de instrumentos confeccionados en una roca cuya procedencia podría estar situada a más de $200 \mathrm{~km}$ del yacimiento. Para abordar este estudio se llevaron a cabo estudios tecno-morfológicos y funcionales sobre los artefactos que permitieron determinar los procesos de confección y uso de este conjunto dentro de la cadena operativa. Por otra parte, el análisis de las posibles fuentes de aprovisionamiento nos permite discutir sobre el rol de estos recursos dentro de los circuitos de movilidad e intercambio de las sociedades cazadoras-recolectoras.
\end{abstract}

PALABRAS CLAVE: cazadores-recolectores, materias primas alóctonas, organización tecnológica, Tierra del Fuego.

\section{DISTANT RAW MATERIALS. EXPLOITATION OF NON-LOCAL LITHIC RESOURCES FOR MICRO-SCRAPERS MANUFACTURE IN THE CENTER OF TIERRA DEL FUEGO: THE CASE OF KAMI 1 ARCHAEOLOGICAL SITE}

\begin{abstract}
The objective of this paper is to present the results obtained from the study of a set of micro endscrapers from the Kami 1 archaeological site, located on the south shore of Fagnano Lake. This assemblage presents as an outstanding feature, a high percentage of instruments made in a rock whose origin could be located more than $200 \mathrm{~km}$ away from the site. In order to undertake this study, we carried out techno-morphological and functional analysis on these artifacts. It was thus possible to determine the processes of production and use of this series of endscrapers within the operative chain. On the other hand, the analysis of possible supply sources allows us to discuss the role of these resources within the circuits of mobility and exchange of hunter-gatherer societies.
\end{abstract} del Fuego.

KEY WORDS: hunter-gatherers, raw materials, allochtonous, technological organization, Tierra 


\section{INTRODUCCIÓN}

El análisis de los conjuntos líticos arqueológicos ofrece una cantidad importante de información acerca de los grupos que los confeccionaron, en particular en lo que se refiere a la organización tecnológica y la gestión de recursos, pero también en cuanto a diversos aspectos de la dinámica social. Así, aspectos tales como explotación de fuentes de materias primas líticas, procesamiento, técnicas y tecnologías asociadas a las diversas características de las materias primas, por ejemplo en relación a la calidad para la talla (Aragón y Franco 1997), pueden informarnos sobre aspectos de la movilidad o la existencia de intercambio en las sociedades en relación a la explotación o uso de estos y otros recursos (Bamforth 1986, Binford 1978 b, 1979 y 1980; Bleed 1986; Gamble 1986; Kelly 1988; Nelson 1984, 1991; Shott 1986; Torrence 1983).

Un aspecto que queremos destacar en el presente trabajo es el análisis de la gestión de las materias primas líticas. Ello implica, entre otras cosas: 1) la búsqueda y el aprovechamiento de las fuentes de aprovisionamiento primarias y secundarias sean locales o no locales (como por ejemplo fuentes distantes); 2) la forma en que ingresan estos recursos al espacio ocupado en aquel momento y cómo son utilizados y descartados dentro del mismo; 3) si la selección de una materia prima específica puede influir o no sobre las técnicas de talla a emplear, y 4) el modo en que esto, finalmente, influirá en el resultado final de la cadena operativa, el instrumento terminado y los desechos de talla generados por su manufactura (Geneste 1985; Karlin 1984, 1991a, 1991b; Leroi-Gourhan 1964; Pélegrin 1984, 1990; Perles 1987).

Para discutir aspectos referidos a la gestión de los recursos líticos, presentaremos el caso de la zona central de la Isla Grande de Tierra del Fuego, y en particular el sitio arqueológico Kami 1 (Mansur et al. 2010; Mansur y De Angelis 2012). Dicho sitio se encuentra ubicado en una zona elevada sobre la margen sur del lago Fagnano $\left(54^{\circ} 35^{\prime} 56.73^{\prime \prime} \mathrm{S}\right.$ y $\left.67^{\circ} 59^{\prime} 47.84^{\prime \prime} \mathrm{O}\right)$. Se trata de un sitio extenso que fue descubierto en el transcurso de prospecciones en la zona, realizadas en el marco del Proyecto Arqueológico Corazón de la Isla (CONICET y Prov. de Tierra del Fuego). Este presentaba zonas con materiales arqueológicos expuestos por procesos erosivos inducidos por el decaimiento del bosque y el paso de turistas, por lo cual se encararon trabajos de campo tendientes a su estudio (proyecto PICT 1236, Sociedad y ritual: Investigaciones arqueológicas en el Corazón de la Isla, dirigido por la Dra. M.E. Mansur). Para su abordaje, el sitio fue íntegramente sectorizado en cuadrículas de dos metros de lado, que permitieron establecer los sectores de excavación y además llevar a cabo la recolección superficial del material arqueológico en aquellos sectores que quedaban expuestos al pisoteo de caminantes y en aquellos que se encontraban en el borde del acantilado.

Kami 1 se caracteriza por la presencia de abundante material lítico, superando las 7500 piezas de las cuales 1171 corresponden a elementos mayores de $2 \mathrm{~cm}$, representado por lascas $(\mathrm{n}=751)$, fragmentos $(n=370)$, raederas $(n=33)$ (Figura 5), raspadores $(n=14)$, fragmentos de instrumentos $(\mathrm{n}=36)$, fragmentos de punta de proyectil, que suman un total de 3 puntas ( $\mathrm{n}=7$ ), y solo un instrumento compuesto. Los núcleos enteros $(n=4)$, núcleos fragmentados $(n=24)$ representando caso el $2 \%$. Mientras que los elementos menores a $2 \mathrm{~cm}$ alcanzan un $\mathrm{n}=6234$ representados por lasquitas $(n=2589)$, debris $(n=2126)$, microlascas $(n=760)$ y microfragmentos $(n=738)$.

Durante la excavación se detectaron al menos tres áreas de combustión asociadas a este material, lo que permitió recuperar una gran cantidad de carbón que permitió fechar al sitio por el método de radiocarbono, el cual arrojo los siguientes fechados: $3210 \pm 80$ AP (LP 2164) y $1130 \pm 60$ AP (LP 2163). Para ver si se trataba realmente de dos episodios diferentes, se decidió realizar un nuevo fechado a partir de carbones del interior del fogón, con el siguiente resultado: $1170 \pm 60 \mathrm{AP}$ (LP 2201), lo que podría estar sugiriendo eventos independientes de ocupación. Sin embargo, los restos faunísticos son muy escasos, probablemente como resultado de la mala preservación del material óseo en sedimentos ácidos como los que se encuentran en los bosques fueguinos.

\section{MATERIAS PRIMAS LÍTICAS EN LA ZONA CENTRAL DE TIERRA DEL FUEGO}

En términos generales, cuando se trata de analizar la gestión de los recursos, y en particular de los líticos por parte de sociedades cazadoras- 
recolectoras, se ha generalizado el uso de dos categorías en relación con la distancia a la que se encuentran las fuentes de provisión de los recursos líticos. Una es la de materias primas locales, para designar aquellas que provienen de fuentes primarias o secundarias que se encuentran a poca distancia del yacimiento y por ello su explotación no implica grandes gastos de energía y tiempo (Church 1994; Luedtke 1979; Nami 1992; Olausson 1982). En contraposición, otra es la de aquéllas que podemos denominar no locales o alóctonas, donde los gastos de energía y tiempo son más altos principalmente debido a que se encuentran a distancias mayores (Charlin 2002; Demars 1982, 1991; Franco y Aragón 2002, 2004; Flegenheimer y Bayón 2002; Franco y Cirigliano 2009; Meltzer 1989; Renfrew 1977).

En cuanto a las materias primas, las investigaciones arqueológicas en la zona central de Tierra del Fuego han indicado que las dos fuentes principales de materias primas son las rocas provenientes de la Formación Lemaire (del Jurásico) y las de la Formación Yaghán (Cretácico) (Caminos 1980; Caminos et al. 1981; Terradas 1995, 1996).

Por la dificultad que a veces revisten para su determinación precisa a ojo desnudo, desde el punto de vista arqueológico estas rocas han sido clasificadas en dos grupos: metamorfitas y vulcanitas (Mansur-Franchomme et al. 1987, Orquera y Piana 1986, Terradas 1996). Entre las primeras, las más utilizadas en los contextos arqueológicos son riolitas y cineritas. Si bien no siempre es fácil distinguirlas a simple vista, estas presentan diferencias en varios aspectos, pero el principal es su granulometría. Las riolitas tienen vitroclastos entre 2 y $0,065 \mathrm{~mm}$, mientras que los asociados a las cineritas son inferiores a $0,065 \mathrm{~mm}$. Las primeras son más sódicas y las segundas más potásicas.

Las vulcanitas de la Formación Yaghan son rocas metamórficas, principalmente pizarras de color negro y grisáceo con una estructura bandeada y una laminación transversal muy marcada (Caminos 1980; Caminos et al. 1981, Terradas 1995, 1996), cuya calidad para la talla es baja por lo que su uso para producción de artefactos es escasa (Terradas 1995, 1996).

También el cuarzo es una materia prima fuertemente representada en diversos contextos arqueológicos. Éste aparece en filones pero tam- bién en formaciones redepositadas como pequeños rodados que pueden ser explotados mediante aplicación de técnica de apertura bipolar (Mansur y Lasa 2005).

El uso de materias primas alóctonas o no locales queda evidenciado en los conjuntos líticos de diversos sitios arqueológicos tanto del norte como del sur de la isla Grande de Tierra del Fuego. Tal es el caso de la obsidiana verde proveniente del Seno de Otway (Morello et al. 2001, Morello et al. 2004), que aparece en sitios como Ma 13 y otros, en la zona de Marazzi (Morello et al. 2012), Aviles 3 (Santiago 2009), al norte de la isla y Túnel I e Imiwaia en la zona del canal Beagle, al sur de la Isla (Orquera y Piana 1999), entre otros.

Para el caso que se presenta en este trabajo, el conjunto lítico posee un grupo conformado por una materia prima que se diferencia del resto y que, aunque faltan estudios por realizar (de tipo geoquímicos, etc.), podrían pertenecer al afloramiento ubicado a más de $200 \mathrm{~km}$ de distancia al norte de la isla. Nos referimos al afloramiento primario de toba silicificada perteneciente a la Formación Palomares del Terciario Superior (Borrazzo 2009; Borrazzo et al. 2010; Prieto et al. 2004), localizado en el valle del chorrillo Miraflores ubicado en territorio chileno al oeste de las nacientes del río Cullen. Esta materia prima se encuentra representada en varios sitios arqueológicos del norte de la isla, aunque en bajas proporciones (Borrazzo 2012; Borrazzo et al. 2010; Massone 2009; Prieto et al. 2004). Recientemente la toba silicificada Miraflores ha sido registrada en lago Blanco, Chile (Morello et al. 2012).

Desde un punto de vista arqueológico, dicha materia prima se caracteriza por presentar una muy buena calidad para la talla, dado que está compuesta por una matriz de grano muy fino que muestra una fractura de tipo concoidea. Sin embargo, esta calidad para la talla se restringe a pequeñas porciones de la roca (Borrazzo 2012; Borrazzo et al. 2010). El color predominante es el gris plomizo (Borrazzo et al. 2010; Prieto et al. 2004).

Dentro del conjunto lítico presentado aquí, esta toba silicificada no local ocupa un lugar preponderante entre los raspadores, ya que más del $60 \%$ de los mismos están confeccionados con esta materia prima. 


\section{EL CASO DE KAMI 1-SITIO 1}

Como punto de partida tomamos los materiales provenientes de las excavaciones realizadas en la localidad arqueológica Kami 1- sitio 1, específicamente el conjunto de raspadores.

La localidad Kami 1 se encuentra ubicada en la margen sur del lago Fagnano, entre las lagunas Bombilla y Palacios. Las lagunas están encerradas por espigas que no llegan a unirse completamente, $y$ es sobre estas geoformas que se encuentra emplazado el sitio (Fig. 1). El sitio 1 se encuentra hacia el interior del bosque, sobre una barranca no muy alta de la costa del lago Fagnano. La playa ubicada por debajo está constituida por guijarros de diversos tamaños y tipos de rocas (riolita, esquisto, cuarzo, etc.) producidos por arrastre glaciario. Dadas las características de la playa, tales como su fácil acceso, la gran cantidad de rodados de tamaños diversos, que está muy cercana al bosque, etc. creemos que estos lugares pudieron ser puntos de convergencia y de uso reiterado por las sociedades pasadas (Mansur et al. 2010).

Los materiales arqueológicos están representados casi en su totalidad por artefactos líticos, y en menor número por fragmentos óseos de los cuales sólo uno supera los $2 \mathrm{~cm}$. El conjunto lítico supera las 7500 piezas, y muestra de forma casi completa la cadena operativa de confección de instrumentos, ya que se identificaron núcleos, lascas de descortezamiento, de adelgazamiento bifacial, desechos de talla e instrumentos terminados y abandonados aún con filos activos. Entre los instrumentos retocados se recuperaron raederas (25 enteras y 20 fragmen-
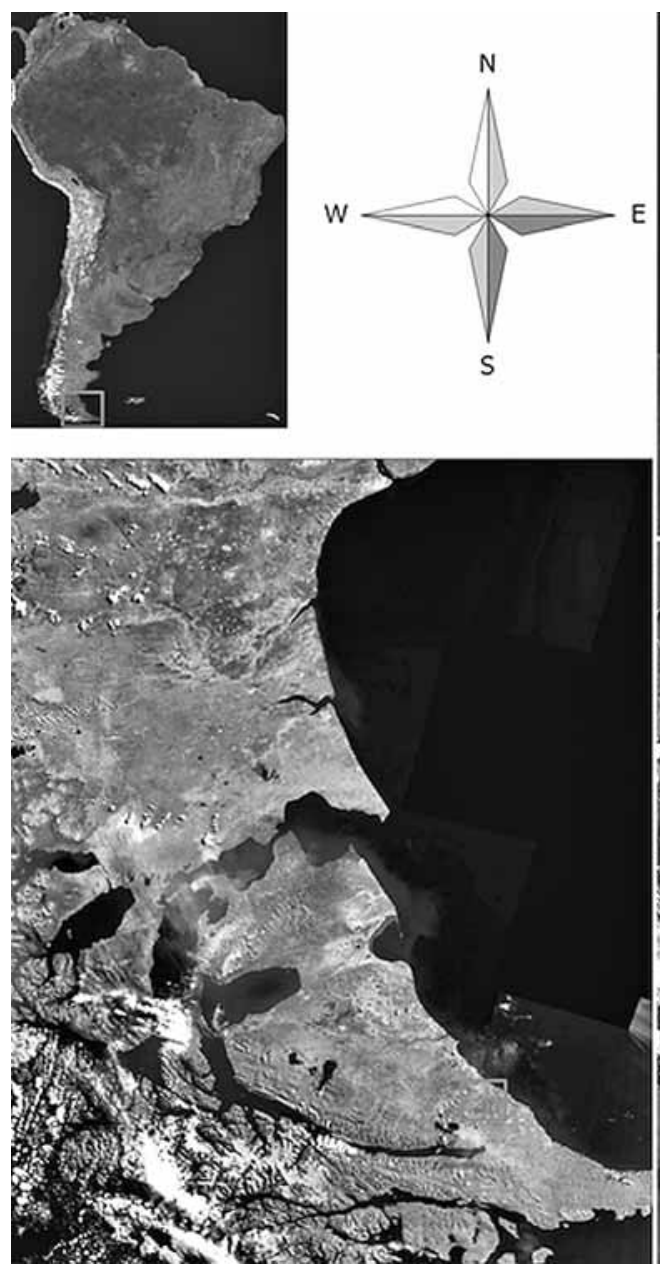

Fig. 1. Ubicación de la localidad arqueológica Kami 1- sitio (imagen obtenida del Google Earth).
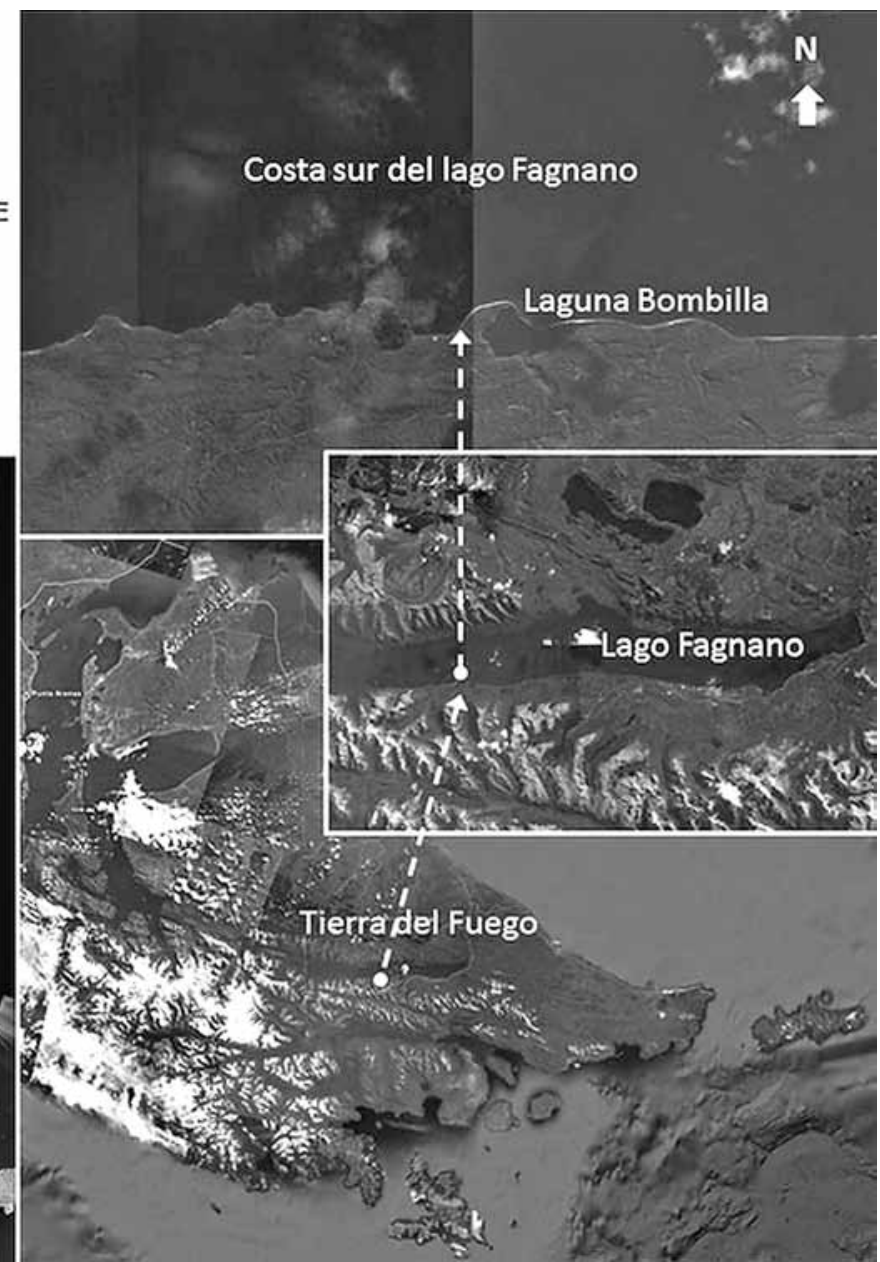

s 

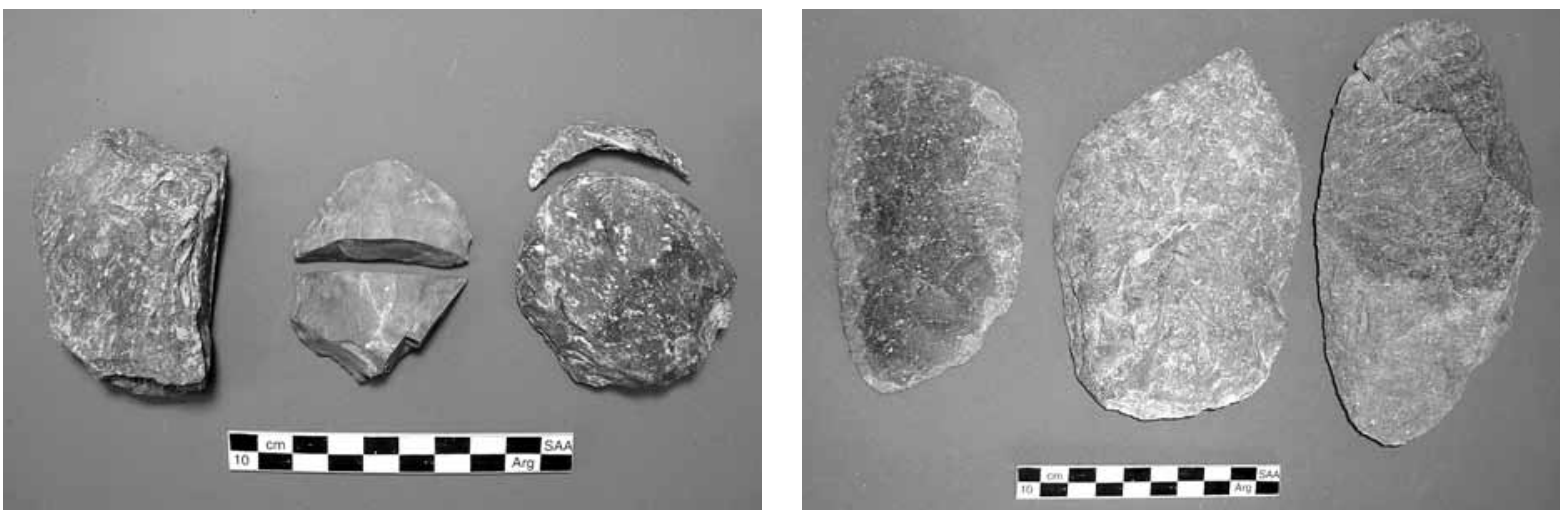

Fig. 2. a ejemplo de raederas, b ejemplo de núcleos. Todos confeccionados sobre riolitas.

tadas), raspadores (33), fragmentos de punta (7) y otros restos (40) que por su grado de fragmentación no pudieron ser determinados (Fig. 2: a y b).

Hasta el momento se contabilizaron 748 lascas, incluidas lascas bipolares de cuarzo y 395 fragmentos mayores a $2 \mathrm{~cm}$ y un total de 6234 elementos menores a $2 \mathrm{~cm}$ entre lasquitas, debris, microlascas y microfragmentos.

\section{CONJUNTO DE TOBA SILICIFICADA}

Como mencionamos anteriormente, dentro del conjunto lítico del sitio Kami 1 , un aspecto que llamó la atención fue la presencia de artefactos de toba silicificada Miraflores $(n=39)$. Estos artefactos se caracterizan por presentar tamaños pequeños a muy pequeños, en su mayoría son desechos de talla, alguno de los cuales son bipolares, mientras que

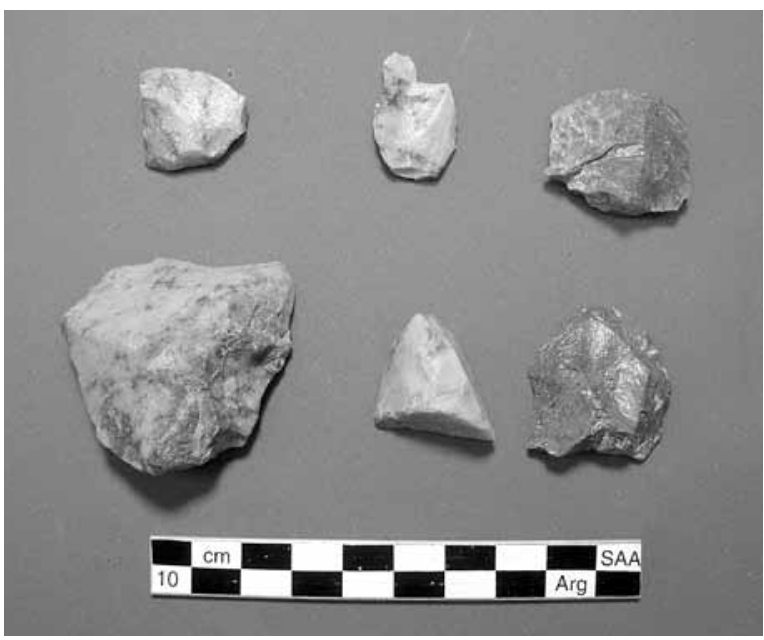

Fig. 3. Diversos artefactos líticos de toba silicificada. otros son resultado de talla por percusión directa. En ninguno de los casos se recuperaron restos de talla con corteza. En cuanto a los núcleos, son pequeñas masas bipolares en su mayoría agotadas, a excepción de uno que presenta rastros de talla por percusión directa y cuyo tamaño es de mediano a pequeño. (Fig. 3).

Finalmente, para este sitio en particular, los únicos instrumentos retocados que se confeccionaron con esta materia prima fueron microrraspadores $(\mathrm{n}=$ 19) (Fig. 4), definidos así debido a que la dimensión mayor no superan los $2 \mathrm{~cm}$, no habiéndose encontrado por ende filos largos retocados (raederas, cuchillos), puntas de proyectil, ni restos de talla que pudieran dar cuenta de la confección de este tipo de instrumental.

A continuación se presentara una breve descripción del análisis tecnomorfológico y funcional de los microrraspadores.

\section{Análisis tecno-morfológico}

El $\mathrm{N}$ total de raspadores es de 33 piezas, sin embargo el número de filos asciende a 44. A grandes rasgos podemos mencionar cinco tipos de materias primas representadas por los raspadores: toba silicificada Miraflores, cuarzo, riolita, sílice, cinerita. La más abundante para este tipo de instrumento

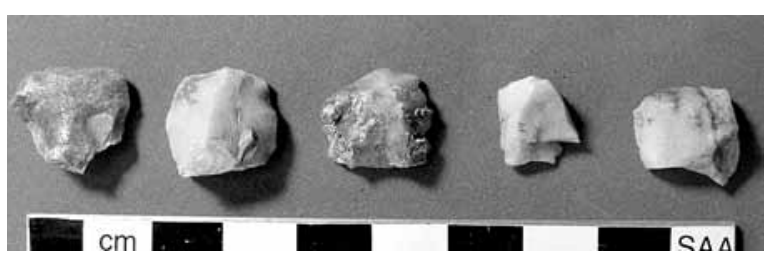

Fig. 4. Raspadores confeccionados sobre toba silicificada. 
es la toba silicificada con 19 piezas), luego el cuarzo con un $\mathrm{n}=6$, riolita y sílice con un 3 artefactos cada una de ellas $y$, finalmente, la cinerita con un $(n=2)$.

Los tipos que incluye el conjunto no presentan una gran diversidad; entre ellos podemos mencionar los raspadores simples $(n=25)$, los atípicos o no estandarizados $(n=4)$, los dobles $(n=3)$ y finalmente un solo raspador triple. El grado de conservación, desde un punto de vista macroscópico, es buena, pudiéndose mencionar un caso de presencia de pátina. El grado de fragmentación es variable, presentándose de la siguiente forma: 22 raspadores enteros, nueve con fracturas, y dos corresponden a fragmentos.

Las dimensiones del conjunto permiten que agrupemos a los raspadores en micro-raspadores (61\%), raspadores chicos (30\%) y raspadores largos (9\%). Para este análisis se tomaron en cuenta los módulos de longitud-anchura y los de ancho-espesor. El primero de ellos arrojó los siguientes resultados: 18 piezas son cortas, 14 medianas y una larga. En el caso del segundo módulo, 20 piezas son gruesas, 12 medianas y una delgada. Estos resultados señalan que se trata de un conjunto de pequeño porte, pero espeso.

Finalmente, en cuanto a la morfología de los raspadores, la forma base presenta las siguientes características: en relación a los soportes sobre los que se confeccionaron los raspadores, se pudo determinar que 20 de ellos se realizaron sobre lascas, ocho a partir de lascas bipolares, cuatro sobre fragmentos y uno sobre una lasca de dorso natural. Los talones son principalmente lisos $(n=14)$, luego se dividen entre facetados y naturales. Cinco raspadores no presentaban talones ya sea que fueron eliminados por talla o fracturas, y sólo en uno se pudo determinar preparación de talón.

De las 17 piezas que presentan bulbo, 12 casos éste es difuso (uno con esquirlamiento) y los cinco restantes corresponden a bulbos espesos. El labio se encuentra escasamente representado ya que sólo nueve instrumentos lo presentan, cinco poseen labio difuso y cuatro labio normal. La corteza está prácticamente ausente, sólo ocho piezas muestran presencia de la misma escasa $(n=4)$; abundante $(\mathrm{n}=3)$ y parcial $(\mathrm{n}=1)^{1}$.

1 Presencia de cortezas sobre la pieza lítica: muy abundante 75 a $100 \%$, abundante 50 a $75 \%$, parcial 25 a $50 \%$ y escasa hasta el $25 \%$.
Descripción de los filos

El total de filos supera al número de raspadores dado que, si bien el conjunto se caracteriza por la presencia de piezas con un solo filo $(n=23)$, también, dentro del conjunto hay nueve piezas con dos filos y una con tres filos retocados, lo que eleva el número de filos a un total de $(n=44)$.

Entre los filos se destacan los distales ( $n=20)$, luego los izquierdos $(n=16)$ y finalmente los derechos $(n=8)$. La delineación muestra un gran representatividad de filos convexos $(n=32)$, le siguen los rectos $(n=7)$ y finalmente los semicirculares $(n=5)$. Los filos en su sección longitudinal son principalmente rectos. Aproximadamente la mitad $(n=22)$ de los filos de los raspadores presentan filos que no superan los 2 centímetros de largo y los $0,3 \mathrm{~cm}$ de alto. Los filos con alturas de 0,4 o superiores poseen longitudes que van desde 0,9 a $3,4 \mathrm{~cm}$.

\section{Descripción del retoque}

El retoque presenta una homogeneidad más marcada que el resto de los atributos antes mencionados. El ángulo es en general intermedio aunque hay algunos rectos. La posición del retoque es directo en 40 de los 44 filos y la extensión es principalmente larga, aunque también hay filos con extensiones restringidas, extendidas y cortas. En todos los casos son filos con retoque continuo y principalmente marginal $(n=36)$, aunque siete presentan una profundidad muy marginal y uno solo, perimetral.

\section{Análisis funcional}

El análisis funcional de base microscópica fue efectuado siguiendo los lineamientos habituales en este tipo de estudio, mediante el uso de una lupa binocular Wild y microscopio óptico de reflexión Olympus BH2, con aumentos comprendidos entre 50 y 500 X, y captura y digitalización de imágenes mediante cámara de videomicroscopía (Mansur 1999; Mansur y Lasa 2005). Mediante este tipo de análisis se pudo determinar que, de los 44 filos, aproximadamente el 35\% presentan rastros de utilización segura ( $\mathrm{N}=15)$. De estos últimos, 13 fueron utilizados sobre piel, uno sobre madera y en un caso no pudo determinarse el material trabajado. En todo los casos, la cinemática fue transversal (raspado). En siete de los filos restantes no pudo determinarse uso, en cuatro de estos casos por alteraciones y en tres 


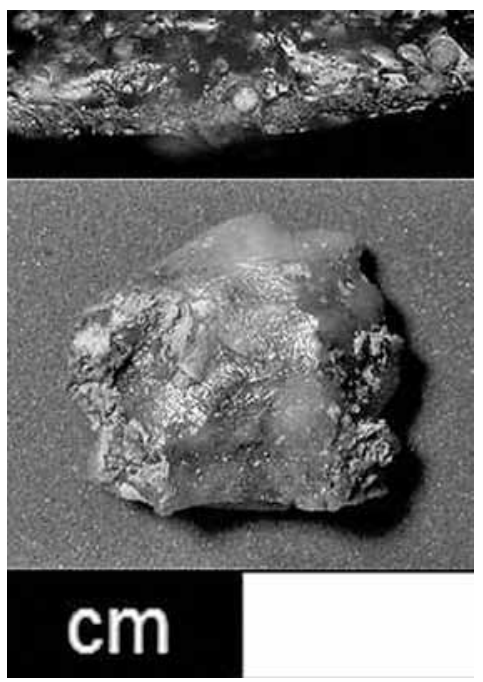

Fig. 5. Arriba microfotografía a 200X de micropulido de cuero. Abajo micro-raspador de toba silicificada.

debido a que el desarrollo de micropulidos no superó el grado 1 de desarrollo (cf. Mansur-Franchomme 1986, Mansur 1999) (Fig. 5).

\section{DISCUSIÓN Y CONCLUSIONES}

Las características del sitio Kami 1, así como de los materiales descubiertos en el transcurso de las excavaciones realizadas, hace que el mismo constituya un buen ejemplo para discutir el tema del aprovisionamiento en materias primas lejanas en Tierra del Fuego. Kami 1, tal como se mencionó anteriormente, se encuentra en la margen sur del lago Fagnano. Una de las características de este sector de la costa es la presencia de una extensa playa de guijarros de diversos tamaños y de diversos tipos de rocas, provenientes tanto de la Formación Yaghan como de la Formación Lemaire. Esta fuente secundaria de materias primas, dada su gran diversidad, presenta un muy alto gradiente de calidades para la talla en los guijarros de la componen. Así tenemos desde rocas con plano de esquistosidad como las pizarras, hasta rocas de granulometría muy fina con fractura concoidea de muy buena calidad para la talla, como es el caso de las cineritas.

Desde este punto de vista se podría decir que estas fuentes de materias primas, ubicadas en lugares puntuales, aunque bastante extensas y muy diversas, ofrecían los recursos necesarios para los grupos que habitaban la zona. Esto también se apoya en la diversidad que muestra el conjunto lítico proveniente del sitio, que se ve tanto en las materias primas como en las técnicas de talla, así como en la diversidad artefactual del conjunto, ya que se pueden reconocer en su totalidad los pasos de la cadena operativa (desde lascas de descortezamiento hasta instrumentos finalizados y utilizados).

Sin embargo, el sitio se singulariza por la presencia de una materia prima diferente, que no se encontró hasta ahora entre los guijarros de la playa del lago. Esta materia prima que se caracteriza por su alta calidad para la talla, de grano muy fino y de color gris plomizo, fue utilizada para la confección de un solo tipo de instrumento, microrraspadores. Se trata de una toba silicificada, que macroscópicamente coincide con la que aflora en fuentes primarias ubicadas a más de $200 \mathrm{~km}$ del lago Fagnano, en el sector norte de Tierra del Fuego (Borrazzo 2010; Borrazzo et al. 2010; Prieto et al. 2004). Este material aparece en el sitio arqueológico bajo la forma de desechos de talla bipolar, lascas producto de talla por percusión directa, pequeños núcleos y finalmente como instrumentos retocados.

Consideramos a esta materia prima como no local, dado que hasta el momento sólo se conoce la fuente ubicada a más de $200 \mathrm{~km}$. La selección y utilización de este material para la confección de raspadores, podría deberse a un alto rendimiento de esta materia prima para efectuar esta tarea, o bien, dado el tamaño de los núcleos trasportados desde el norte de la isla, no permitiría la confección de instrumentos de mayor tamaño, como si lo permitieron las rocas locales con las que se confeccionaron raederas con filos de más de $10 \mathrm{~cm}$. Por el momento descartamos que esta materia prima provenga de los guijarros de la costa del Fagnano debido la falta de lascas con corteza, a diferencia de lo que ocurre con las materias primas restantes, entre las que sí se hallaron gran cantidad de lascas con abundante corteza cuyas características permiten corroborar que pertenecen a guijarros de playa.

Hasta el momento no queda claro si la obtención de este material se relaciona con un ciclo móvil específico y por lo tanto estas fuentes de aprovisionamiento quedaban dentro del mismo, o por intercambio con grupos de más al norte (Borrazzo 2012). Sin embargo, lo que queda evidenciado es la movilidad en relación a la búsqueda de esta materia prima. 
Otro rasgo que llamó la atención dentro del conjunto lítico es la presencia de un gran número de raspadores de muy pequeño tamaño, microrraspadores, los que en su gran mayoría fueron confeccionados en toba silicificada, pero también se utilizaron otras materias primas, en menor medida como por ejemplo riolita, cuarzo, cinerita y sílice. Esta concentración alta de raspadores o microrraspadores sólo se conoce hasta el momento en tres sitios de la zona del canal Beagle, Túnel I (II y IV componente), Imiwaia I y Shamakush I (Álvarez et al. 2000; Mansur y Lasa 2005).

Para el caso del mayor conjunto estudiado, el de los microrraspadores del IV componente de Túnel I, Mansur y Lasa (2005) concluyen que se trata de una selección voluntaria de una materia prima específica, el cuarzo, para una tarea específica también (el raspado de pieles) debido a las características de efectividad y durabilidad de los filos para esta tarea, en contraposición con los de otras materias primas que tienen tendencia al desgranamiento. Lo mismo fue observado en otros sitios de Pampa y Patagonia, por lo cual Mansur propuso que la selección de determinadas materias primas para la confección de raspadores está condicionada por las características de los filos resultantes en función de su utilización (Leipus y Mansur 2007; Mansur 2007; Mansur y Lasa 2005). Para el caso de estudio, no todos los filos ofrecieron resultados positivos en el análisis funcional, pero de los 15 filos que si fueron positivos, 13 de ellos mostraron trabajo transversal sobre cuero, esto significa que en casi su totalidad estos instrumentos fueron utilizados sobre un sólo tipo de material, cuyos requerimientos en cuanto a durabilidad son comparables a los mencionados.

Así, la homogeneidad morfológica del conjunto de micro-raspadores así como su homogeneidad funcional podría estar indicando una búsqueda dirigida hacia un tipo de instrumentos específicos para una tarea específica. Debido al tamaño de estos artefactos es muy probable que hayan sido enmangados para su utilización. Sin embargo el análisis funcional no arrojo resultados positivos que puedan corroborar esta hipótesis.

Sabemos que para momentos históricos, la sociedad Selk'nam tenía territorios definidos, conocidos como Haruwen, que pertenecían a cada familia. Dado que estas fronteras eran muy permeables, las personas podían moverse de unos a otros para visitarse, o para las diversas celebraciones como por ejemplo un Hain (Borrero 1986, 1991; Chapman 1986, 2008; Gusinde 1982; Mansur y Piqué 2009). Estos movimientos pueden ser ocasión de intercambio y movimiento de materias primas, de ideas o conocimientos. Entonces es posible que algo similar pudiera ocurrir en el pasado, al menos hace ca. 1000 años basándonos en los fechados radiocarbónicos, y que esta movilidad hubiera permitido el ingreso del material de la fuente Miraflores.

\section{AGRADECIMIENTOS}

Mauricio Gonzales Guillot, Pablo Torres Carbonel por el tiempo y la riqueza que aportaron a la discusión sobre materias primas, Estela Mansur por las correcciones, Vanesa Parmigiani por sus aportes, a Karen Borrazzo, Luis Borrero y Alfredo Prieto por la bibliografía aportada.

\section{BIBLIOGRAFÍA}

ARAGON, E. y N. V. FRANCO. 1997. Características de rocas para la talla por percusión y propiedades petrográficas. Anales del Instituto de la Patagonia, Serie Ciencias Humanas 25: 87-199.

ÁlVAREZ, M.R., A.E. LASA Y M.E. MANSUR. 2000. La explotación de recursos naturales perecederos: análisis funcional de los raspadores de la costa norte del Canal Beagle. Relaciones de la Sociedad Argentina de Antropología XXV: 275-296.

BAMFORTH, D. 1986. Technological efficiency and tool curation. American Antiquity 51:38-50.

BINFORD, L. 1978. Dimensional analysis of behavior and site structure: learning from an Eskimo hunting stand. American Antiquity 43(3):330-361.

1979. Organization and formation processes: looking and curated technologies. Journal Anthrop. Research 35(3):255-273.

1980.Willow smoke and Dogs Tails: Hunter-Gatherer settlement systems and archaeological site formation. American Antiquity 45(1):4-20.

BLEED, P. 1986. The optimal design of hunting weapons: maintainability or reliability? American Antiquity 56: $19-35$.

BORRAZZO, K. 2009. El uso prehistórico de los afloramientos terciarios en la bahía San Sebastián (Tierra del Fuego, Argentina). En: Arqueología de Patagonia. Una mirada 
desde el último confín. Editado por M. Salemme, F. Santiago, M. Álvarez, E. Piana, M. Vázquez y M.E. Mansur. Pp. 291-305. Editorial Utopías, Ushuaia. 2010. Arqueología de los esteparios fueguinos. Tesis para optar al grado de doctor en el área de Arqueología. Facultad de Filosofía y Letras, Universidad de Buenos Aires, Buenos Aires. MS.

2012. Raw material availability, flaking quality and hunter-gatherer decisión making in Northern Tierra del Fuego Island (Argentina). Journal of Archaeological Science, en prensa. http://dx.doi.org/10.1016/j. jas.2012.03.018.

BORRAZZO, K., M., D'ORAZIO, Y M. C., ETCHICHURY. 2010. Distribución Espacial y Uso Prehistórico de las Materias Primas Líticas del Chorrillo Miraflores en el Norte de la Isla Grande de Tierra del Fuego (Argentina). Revista Chilena de Antropología 22:77-97.

BORRERO, L. A. 1986. La economía prehistórica de los habitantes del norte de la Isla Grande de Tierra del Fuego. Tesis doctoral, Facultad de Filosofía y Letras, Universidad de Buenos Aires. MS.

1991. Los Selk'nam (Onas). Su Evolución Cultural. Ed. Búsqueda-Yuchán, Buenos Aires.

CAMINOS, R.1980. Cordillera Fueguina. Geología Regional Argentina 2:1463-1501. Academia Nacional de Ciencias. Córdoba.

CAMINOS, R., M., HALLER, O., LAPIDO, A., LIZUAIN, R., PAGE, V., RAMOS. 1981. Reconocimiento geológico de los Andes Fueguinos. Territorio Nacional de Tierra del Fuego. En Actas del VIII Congreso Geológico Argentino III, pp.759-786, San Luis.

CHAPMAN, A. 1986. Los Selk'nam. La vida de los Onas. Emecé editores, Buenos Aires.

2008. End of a Word: The selknam of Tierra del Fuego. Zagier \& Urruty, Ushuaia.

CHARLIN, J. 2002. Aprovisionamiento de materias primas líticas en el NO de la provincia de La Pampa a fines del siglo XIX. En: Del Mar a Los Salitrales. 10000 años de historia pampeana en el umbral del tercer milenio. 205 - 218. Editado por Mazzanti, D., M. Berón y F. Oliva. Universidad Nacional de Mar del Plata y Sociedad Argentina de Antropología, Mar del Plata.

$\mathrm{CHURCH}, \mathrm{T} .1994$ Terms in lithic resource studies. Lithic resource studies: a source for archaeologist. Lithic Technology 3: 9-25.

DEMARS P.Y. 1982. L'utilisation du silex au Paléolithique Supérieur: choix, approvisionnement, circulation. L'exemple du bassin du Brive. En: Cahiers du Quaternaire nº 5, CNRS, Bordeaux.
DEMARS, P.Y. 1994. L'économie du silex au Paléolithique supérieur dans le Nord de l'Aquitaine. Tesis de Doctorado presentada en la Universidad de Bordeaux I. Francia.

FLEGENHEIMER N., Y C. BAYÓN. 2002. ¿Cómo, cuándo y dónde? Estrategias de abastecimiento lítico en la pampa bonaerense. En: Del Mar a Los Salitrales. 10000 años de historia pampeana en el umbral del tercer milenio. 231-241. Editado por Mazzanti, D., M. Berón y F. Oliva. Universidad Nacional de Mar del Plata y Sociedad Argentina de Antropología, Mar del Plata.

FRANCO N.V. Y E. ARAGÓN. 2002. Muestreo de fuentes potenciales de aprovisionamiento lítico: un caso de estudio. En: Del Mar a Los Salitrales. 10000 años de historia pampeana en el umbral del tercer milenio. 243-250. Editado por Mazzanti, D., M. Berón y F. Oliva,. Universidad Nacional de Mar del Plata y Sociedad Argentina de Antropología, Mar del Plata.

2004. Variabilidad en fuentes secundarias de aprovisionamiento lítico: El caso del sur del lago Argentino (Santa Cruz, Argentina). Estudios Atacameños 28:71-85.

FRANCO N. Y N. CIRIGLIANO. 2009. Materias primas y movilidad humana entre las cuencas de los ríos Santa Cruz y Chico (provincia de Santa Cruz, Argentina): primeros resultados. En Arqueología de Patagonia: Una mirada desde el último confín, 361-368. Editado por M. Salemme, F. Santiago, M. Álvarez, E. Piana, M. Vázquez y M.E. Mansur. Editorial Utopías, Ushuaia.

GAMBLE, C. 1986. The paleolithic settlement of Europe. Cambridge University Press, Cambridge.

GENESTE, J.M. 1985. Analyse lithique d'industries moustériennes du Périgord: une approche technologique $d u$ comportement des groupes humains au Paléolithique moyen. Tesis de doctorado, Université Bordeaux I. MS.

GUSINDE, M. 1982 [1937]. Los indios de Tierra del Fuego. Tomo 1: Los Selk'nam. 2 vols. Centro Argentino de Etnología Americana, Buenos Aires.

KARLIN, C. 1984. Le débitage du silex à Pincevent, précedé de quelques notes de vocabulaire. En: Tixier, J. (Ed.): Prehistoire de la Pierre taillée 2. Économi du débitage laminaire. CNRS, pp. 39-44. Paris.

1991a. Connaissences et savoir-faire: comment analyser un processus technique en Préhistoire: Introduction. En: Tecnología y cadenas operativas líticas. Mora, R; X. Terradas, A. Parpal y C. Plana (Eds.), pp. 99-124. Bellaterras, Barcelona.

1991b. Analyse d'un processus technique: le débitage laminaire des magdaleniens de Pincevent (Seine et Marne). En: Tecnología y cadenas operativas líticas. Mora, R; 
X. Terradas, A. Parpal y C. Plana (Eds.), pp.125-162. Bellaterras, Barcelona.

KELLY, R. 1988. The three sides of a bifaces. American Antiquity 53 (4):717-734.

LEIPUS, M. y M.E. MANSUR. 2007. El análisis funcional de base microscópica aplicado a materiales heterogéneos. Perspectivas metodológicas para el estudio de las cuarcitas de la Región Pampeana. En: Arqueología de las Pampas. Tomo I. Pp 179-200. Sociedad Argentina de Antropología. Buenos Aires.

LEROI-GOURHAN, A. 1964. Le Geste et la Parole I. Technique et langage. Albin Michel, Paris.

LUEDTKE, B. 1979. The identification of sources of chert artifacts. American Antiquity 44:744-756.

MANSUR, M. E. 1999. Análisis de instrumental lítico: problemas de formación y deformación de rastros de uso. Actas del XII Congreso Nacional de Arqueología Argentina (1):355-366. La Plata.

2007. Arqueología de la zona de Punta Bustamante (Prov. de Santa Cruz, Argentina). En: Arqueología de la costa Patagónica. Perspectivas para la conservación. I. Cruz y M. S. Caracotche. (Edis), UNPA. Pp. 173-193.

MANSUR-FRANCHOMME, M.E. 1986, Microscopie du materiel préhistorique : traces d'utilisations naturelles, accidentelles et technologiques. Cahiers du Quaternarie 9, CNRS, Bordeaux, Francia.

1987. Outils ethnographiques de Patagonie. Emmanchement et traces d'utilisation. En: La Main et l'outil. Manches et emmanchements préhistoriques. 297-307. Travaux de la Maison de l'Orient 15, Lyon.

MANSUR, M.E. y A. LASA. 2005. Diversidad artefactual vs. especialización funcional. Análisis del IV componente de Túnel I (Tierra del Fuego, Argentina). Magallania 33 (2): 69-91. Universidad de Magallanes, Punta Arenas, Chile.

MANSUR, M. E. y R. PIQUÉ. 2009. Between the forest and the sea: hunter-gatherer occupations in the subantarctic forests in Tierra del Fuego (Argentina). Arctic Anthropology 46 (1-2): 144-157.

MANSUR M. E., H. DE ANGELIS y V. PARMIGIANI. 2010. Explotación de materias primas y circuitos de movilidad en la zona central de Tierra del Fuego. En: Arqueología Argentina en el Bicentenario de la Revolución de Mayo. Actas del XVII Congreso Nacional de Arqueología Argentina, tomo V: 1935-1940. Mendoza.

MANSUR, M.E. y H. DE ANGELIS. 2012. Ambientes, recursos y dinámica poblacional en la faja central de Tierra del Fuego: el bosque sub-antártico. En: Tendencias teóricometodológicas y casos de estudio en la arqueología de Patagonia. En prensa.
MASSONE, M. 1997. Prospección Arqueológica del Sector comprendido entre los ríos Marazzi y Torcido, zona norte de Tierra del Fuego. Anales del Instituto de la Patagonia, Serie Ciencias Humanas, 25:123-136.

2009. Territorios, movilidad y construcción de paisajes en el mundo selk'nam. En: Arqueología de Patagonia: Una mirada desde el último confín, editado por $\mathrm{M}$. Salemme, F. Santiago, M. Álvarez, E. Piana, M. Vázquez y M. Mansur, 249-264. Editorial Utopías. Ushuaia.

MELTZER, D. 1989. Was Stone Exchange Among Eastern North American Paleoindians? En: Eastern Paleoindian lithic resource use, Ed. C. Ellis y J. Lothrop, 11-39. Westview Press, Boulder.

MORELLO, F., M. SAN ROMÁN, A. PRIETO Y C. STERN. 2001. Nuevos avances para una discusión arqueológica en torno a la obsidiana verde en Patagonia Meridional. Anales del Instituto de la Patagonia, Serie Ciencias Humanas 29: 129-148.

MORELLO, F., M. SAN ROMÁN, Y A. PRIETO. 2004. Obsidiana verde en Fuego-Patagonia: distribución y estrategias tecnológicas, Civalero (Ed.). En: Contra Viento y Marea. Arqueología de Patagonia: 149-166. Buenos Aires: Edición del INAPL y la SAA.

MORELLO, F., BORRERO, L.A., MASSONE, M., STERN, C., GARCIA-HERBST, A., MCCULLOCH, R., ARROYO KALIN, M., CALAS, E., TORRES, J., PRIETO, A., MARTÍNEZ, I. BAHAMONDE, G., CÁRDENAS, P. 2012. Hunter-gatherers, biogeographic barriers and the development of human settlement in Tierra del Fuego. Antiquity 86: 71-87.

NAMI, H.G. 1992. El subsistema tecnológico de confección de instrumentos líticos y explotación de los recursos del ambiente: Una nueva vía de aproximación. Shincal 2:33-53.

NELSON, M. 1984. Ladder Ranch Resarch Project: first season. Maxwell University of New Mexico Occasional Papers 1. Albuquerque.

1991. The study of technological organization. Archaeological Method and Theory, Vol. 3. M. Schiffer, Ed. University of Arizona Press.

OLAUSSON, D. 1982. Flint and groundstone axes in the Scanian Neolithic. An evaluation of raw materials based on experiment. Scripta Minora, 2, Lund, Suecia.

ORQUERA, L. Y E. PIANA. 1986. Normas para la descripción de objetos arqueológicos de piedra tallada. Contribución científica 1. CADIC. Ushuaia. 1999. Arqueología de la Región del Canal Beagle Tierra del Fuego, República Argentina. Buenos Aires: Sociedad Argentina de Antropologia. 
PELEGRIN, J. 1990. Préhistoric lithic technology: some aspects of research. Archeologucal Review, from Cambridge, 9, pp.116-125.

1984, Approche technologique expérimentale de la mise en forme de nucléus pour le débitage systématique par presión. En Tixier, J. (ed.) Prehistoire de la Pierre taillée 2. Économie du débitage laminaire. París, CNRS, pp. 93-103.

PERLES, C. 1987. Les industries lithiques taillées de Franchthi (Argolide, Gréce). Présentation genérale et industries paléolihiques. Excavations at Franchthi Cave-GreeceEditado por T. W. Jacobsen. Indianana University Press. Bloomington. Indianapolis.

PRIETO, A., P. CÁRDENAS, G. BAHAMONDE Y M. MASSONE. 2004. Hallazgo de una fuente de materia prima en el chorrillo Miraflores, Tierra del Fuego, Chile. Magallania 32: 229-232.

RENFREW, C. 1977. Alternative models for exchange and spatial distribution. En: Exchange Systems in Prehistory, Ed. T. Earle y J. Ericson, 71-90. Academic Press, New York.
SANTIAGO, F. 2009. La ocupación humana del norte de Tierra del Fuego durante el Holoceno medio y tardío. Su vinculación con el paisaje. Tesis Doctoral. Universidad Nacional del Centro, Olavarría, Argentina.

SHOTT, M.1986. Technological organization and settlement mobility: an ethnographic examination. Journal of Anthropological Research 42: 15.

TERRADAS, X. 1995. Lithic raw material procurement strategies of the Yamana people (Tierra del Fuego, Argentina). En: Man and Flint (R. Schild and Z. Sulgostowska, eds.). Varsovia: Institute of Archaeology and Ethnology. 1996. La gestió dels recursos minerals entre les comunitatas caçadores-recol-lectores. Vers una representació de les estratègies de proveïment de matèries primeres. Tesis de Doctorado. Departement d'Historia de les Societats Precapitalistes i d' Antropologia Social. Universitat Autonoma de Barcelona. MS.

TORRENCE, R. 1983. Time Budgeting and Hunter-Gatherer Technology. Hunter-Gatherer economy in Prehistory: a european perspective. Editado por G. Bailey, 11-22. Cambridge University Press, Cambridge. 
